\title{
Signatures of Molecular Orbital Structure in Lateral Electron Momentum Distributions from Strong-Field Ionization
}

\author{
Ingo Petersen, Jost Henkel, and Manfred Lein \\ Institut für Theoretische Physik and Centre for Quantum Engineering and Space-Time Research (QUEST), \\ Leibniz Universität Hannover, Appelstraße 2, 30167 Hannover, Germany
}

(Received 3 December 2014; published 13 March 2015)

\begin{abstract}
Strong-field ionization of aligned diatomic and polyatomic molecules such as $\mathrm{O}_{2}, \mathrm{~N}_{2}, \mathrm{C}_{2} \mathrm{H}_{4}$, and others in circularly polarized laser fields is investigated theoretically. By calculating the emission-angle-resolved lateral width of the momentum distribution perpendicular to the polarization plane, we show that nodal planes in molecular orbitals are directly imprinted on the angular dependence of the width. We demonstrate that orbital symmetries can be distinguished with the information obtained by observing the lateral width in addition to the angular distributions.
\end{abstract}

DOI: 10.1103/PhysRevLett.114.103004

PACS numbers: $33.80 . \mathrm{Rv}, 33.20 . \mathrm{Xx}$

Progress in strong-field science has led to the possibility to image dynamics in atoms and molecules on the attosecond time scale and angstrom spatial scale [1-3]. Laser-based methods to image molecular structure include diffraction of recolliding electrons [4-6], Coulomb explosion imaging [7,8], and orbital tomography [9-14]. Information about the orbital structure of aligned molecules is also contained in the dependence of the ionization rate on the angle between the laser polarization direction and the molecular axis $[15,16]$ with particular care needed for polar molecules [17]. Rich information is obtained by measuring three-dimensional photoelectron momentum distributions (PMDs) of aligned molecules. This is possible by combining laser-induced alignment of molecules [18,19] and velocity-map imaging [20,21]. Alternatively, PMDs can be measured in a reaction microscope [22], where information on the molecular orientation is obtained by electron-ion coincidence. For molecular imaging, circularly polarized light effectively delivers a $360^{\circ}$ scan of the aligned molecule [23-25], which is an advantage over linear polarization. In the classical trajectory model without Coulomb effects, electrons measured at an emission angle $\phi$ originate from ionization at the angle $\phi \pm 90^{\circ}$ with the sign depending on the rotation direction of the field. Another advantage of circular polarization is the absence of electron recollisions that could obscure the measured distribution. Nodal structures have been measured successfully for various molecules using many-cycle circularly polarized pulses [26-29]. Theoretical prediction of the PMDs is complicated by the nonperturbative nature of the ionization process in the commonly used near-infrared fields. Only for very small molecules such as $\mathrm{H}_{2}{ }^{+}$[30] or in proof-of-principle calculations with model orbitals [31], the solution of the timedependent Schrödinger equation (TDSE) is an option. In contrast, the strong-field approximation (SFA) can be used to calculate PMDs of arbitrary molecules and often yields accurate results [32-34]. In polar molecules, an extended SFA including the Stark shift is beneficial [35-37].
To the authors' knowledge, none of the published experimental work on molecular imaging has made use of the lateral photoelectron momentum distribution (LPMD), i.e., the distribution of the momentum component perpendicular to the plane of polarization. Recent experiments have demonstrated the possibility to measure the angle dependence of the width of the LPMD [38]. For atoms in the deep tunneling regime, the LPMD is well approximated by a product form, where one factor is a Gaussian [39] and the other is directly related to the orbital from which ionization takes place [40]. The lateral width follows the ionizing field on a subcycle time scale and is much more robust against Coulomb and depletion effects than the angular distribution itself [41]. Hence, the LPMD is a promising tool for molecular imaging.

In this Letter, we calculate the angle dependence of the LPMD for various molecules in circular polarization and we compare it to the angular distribution of the electron yield. We demonstrate that the molecular structure is clearly imprinted onto the LPMD. We use the SFA [42-44] in length gauge to compute PMDs (in atomic units)

$w(\mathbf{k})=\int_{0}^{2 \pi} d \xi\left|\int_{T_{1}}^{T_{2}} d t\left\langle\mathbf{k}+\mathbf{A}(t)|\mathbf{r} \cdot \mathbf{E}(t)| \psi_{0}^{\xi}\right\rangle e^{i S(t)}\right|^{2}$

for ionization of aligned molecules. In Eq. (1), $S(t)=$ $I_{p} t+(1 / 2) \int^{t} d \tau[\mathbf{k}+\mathbf{A}(\tau)]^{2}$ is the action, $I_{p}$ is the ionization potential, and the laser electric field $\mathbf{E}(t)=-\dot{\mathbf{A}}(t)$ lies in the $x y$ plane. The continuum states $|\mathbf{k}+\mathbf{A}(t)\rangle$ are approximated as plane waves. The molecules are aligned with their axis along the $x$ direction of the laboratory frame, and the angle $\xi$ describes a rotation of the molecule about its axis. Hence, the integration $d \xi$ indicates averaging over all molecular orientations compatible with one-dimensional alignment. We include only the highest occupied molecular orbital (HOMO) $\psi_{0}^{\xi}$, obtained from quantum chemistry software with the Becke three-parameter Lee-Yang-Parr 
(B3LYP) method [45,46], except in the case of $\mathrm{H}_{2}{ }^{+}$, where the ground state is found by imaginary-time propagation for a model potential. In the case of a $\sigma$-type orbital, the integration over $\xi$ is equivalent to a multiplication with $2 \pi$. Our model ignores depletion and dynamical correlation, so we apply it to moderate intensities. We use a $800 \mathrm{~nm}$ wavelength and a constant amplitude $E_{0}$ corresponding to an intensity of $I=2.5 \times 10^{14} \mathrm{~W} / \mathrm{cm}^{2}$, unless specified otherwise. Choosing a continuous wave (cw) field has the following reasons: (i) multicycle pulses avoid anisotropies introduced by the pulse envelope and thus bring out the angle-dependent ionization probability of the molecular orbital [24] and (ii) the cw field reduces the numerical workload.

For a short pulse we define the LPMD as

$$
L_{\phi}\left(k_{z}\right)=\int_{0}^{\infty} d \kappa \kappa w\left(\kappa, \phi, k_{z}\right)
$$

for each azimuthal angle $\phi$ measured from the $k_{x}$ axis. Here, cylindrical coordinates $\left(\kappa, \phi, k_{z}\right)$ are used, i.e., $\mathbf{k}^{2}=\kappa^{2}+k_{z}^{2}$. For the cw field, we consider the momentumresolved ionization rate $\tilde{w}(\mathbf{k})=w(\mathbf{k}) /\left(T_{2}-T_{1}\right)$ for $T_{2}-T_{1} \rightarrow \infty$ to calculate the LPMD as

$$
L_{\phi}\left(k_{z}\right)=\int_{0}^{\infty} d \kappa \kappa \tilde{w}\left(\kappa, \phi, k_{z}\right)=(\omega / T) \sum_{n=n_{0}}^{\infty} \tilde{w}_{n}\left(\phi, k_{z}\right),
$$

with

$\tilde{w}_{n}\left(\phi, k_{z}\right)=\int_{0}^{2 \pi} d \xi\left|\int_{0}^{T} d t\left\langle\mathbf{k}_{n}+\mathbf{A}(t)|\mathbf{r} \cdot \mathbf{E}(t)| \psi_{0}^{\xi}\right\rangle e^{i S(t)}\right|^{2}$

and $\mathbf{k}_{n}=\hat{\mathbf{e}}_{z} k_{z}+\sqrt{2\left(n \omega-I_{p}-U_{p}-k_{z}^{2} / 2\right)}\left(\hat{\mathbf{e}}_{x} \cos \phi+\hat{\mathbf{e}}_{y} \sin \phi\right)$. The summation starts from the smallest possible integer $n_{0}$ that gives a non-negative argument of the square root. $U_{p}=E_{0}^{2} /\left(2 \omega^{2}\right)$ is the ponderomotive potential for circular polarization at frequency $\omega=2 \pi / T$. The photoelectron angular distribution (PAD) is the integral over the lateral momentum $k_{z}$ :

$$
P(\phi)=\int d k_{z} L_{\phi}\left(k_{z}\right) .
$$

To quantify the spread of the LPMD, we define the width

$$
\sigma(\phi)=\sqrt{\frac{2 \int d k_{z}\left(k_{z}-\left\langle k_{z}\right\rangle_{\phi}\right)^{2} L_{\phi}\left(k_{z}\right)}{\int d k_{z} L_{\phi}\left(k_{z}\right)}},
$$

where the average lateral momentum $\left\langle k_{z}\right\rangle_{\phi}$ is zero in the cases shown in this Letter. If $L_{\phi}\left(k_{z}\right)$ is a Gaussian, Eq. (6) yields the same value as the definition used previously for atoms [40,47]. In general, the distribution is not always similar to a Gaussian $[48,49]$. Therefore, the definition according to Eq. (6) is more appropriate.
First, we assess the applicability of the SFA in the calculation of the LPMD for molecules. For this purpose, we numerically solve the TDSE

$$
i \partial_{t} \psi(t)=\left(-\frac{\nabla^{2}}{2}+\mathbf{r} \cdot \mathbf{E}(t)+V(\mathbf{r})\right) \psi(t)
$$

for $\mathrm{H}_{2}{ }^{+}$with fixed nuclei and a soft-core potential as in Eq. (4) of Ref. [50] with internuclear distance $R=2$ a.u. Here, we use short laser pulses with electric-field vector

$$
\mathbf{E}_{\varphi}(t)=E_{0} f(t)(\cos (\omega t+\varphi), \sin (\omega t+\varphi), 0)
$$

for $0 \leq t \leq 3 T$ with $f(t)=\sin ^{2}(\omega t / 6)$ and carrierenvelope phase (CEP) $\varphi$. The peak field $E_{0}$ is the same as the cw field used in the SFA. The final momentum wave function $\tilde{\psi}_{f, \varphi}$ of the emitted electron is obtained as in Ref. [51]. The LPMD is computed for each CEP:

$$
L_{\phi, \varphi}^{\mathrm{TDSE}}\left(k_{z}\right)=\int_{0}^{\infty} d \kappa \kappa\left|\tilde{\psi}_{f, \varphi}\left(\kappa, \phi, k_{z}\right)\right|^{2} .
$$

From this distribution, we calculate the lateral width $\sigma_{\varphi}(\phi)$ according to Eq. (6) for each CEP. According to the classical model without Coulomb effects [52], the electrons launched at the time of maximum field, $t=3 T / 2$, acquire the final momentum $-\mathbf{A}_{\varphi}(3 T / 2)$; i.e., they are emitted at the angle $\phi_{\varphi, \max }=\pi / 2+\varphi$. We calculate the yield $P$ and the width $\sigma$ at the angle $\phi_{\varphi \text {,max }}$ for each CEP and thus find these quantities in dependence of the emission angle $\phi_{\varphi, \max }$. To compare short-pulse and cw results, we identify $\phi_{\varphi, \max }$ with the emission angle $\phi$ in the cw calculation. The angle $\phi_{\varphi, \text { max }}$ is not necessarily where the width or the yield is maximal. This is due to competing influences from field envelope and orbital shape and furthermore due to Coulomb effects on the outgoing electron [53].

Figure 1 shows the comparison of the $\mathrm{H}_{2}{ }^{+} \mathrm{cw}$ SFA, shortpulse SFA, and TDSE results. The same initial ground-state wave function is used in all calculations. Since the molecular axis points along the $x$ axis $(\phi=0)$ and the highest ionization probability is expected for fields parallel to this direction, we expect the maximum signal at $\phi=90^{\circ}$.

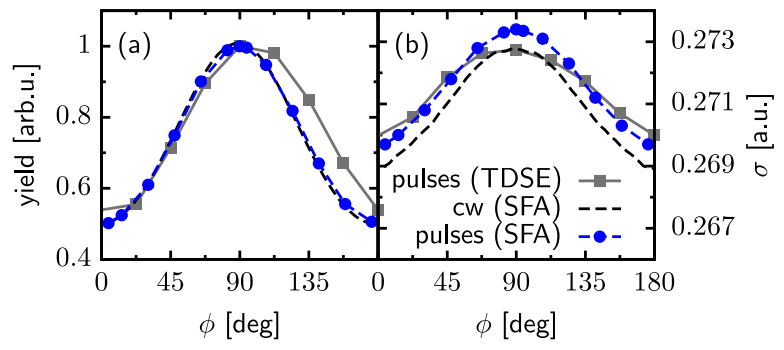

FIG. 1 (color online). (a) Angle-dependent normalized electron yield and (b) lateral width for $\mathrm{H}_{2}{ }^{+}$ionized by a cw field with intensity $8.49 \times 10^{14} \mathrm{~W} / \mathrm{cm}^{2}$ and wavelength $800 \mathrm{~nm}$ (SFA, black dashed curves), and by short pulses with different CEP values (TDSE, gray squares; SFA, blue circles). 


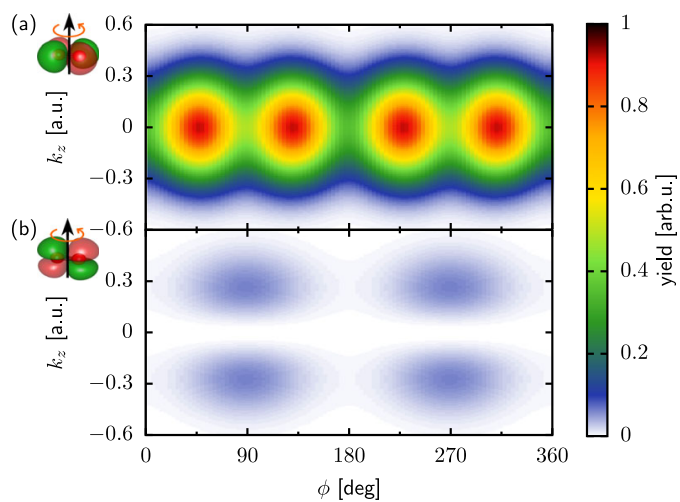

FIG. 2 (color online). Lateral photoelectron momentum distribution $L_{\phi}\left(k_{z}\right)$ according to Eq. (2) for the two degenerate HOMOs of $\mathrm{O}_{2}$, using SFA. (a) $\mathrm{HOMO}_{1}$, (b) $\mathrm{HOMO}_{2}$.

Figure 1(a) shows that this holds only in SFA. In the TDSE, the maximum is shifted towards larger angles, similar to what has been previously found [30]. However, the maximal width is always at $\phi=90^{\circ}$. Such a strikingly different behavior of the two observables has been found already for atoms [41]. As with atoms [40], the widths computed with SFA and TDSE are in very good quantitative agreement. This justifies the use of the SFA. We note that for $\mathrm{H}_{2}{ }^{+}$the width is only weakly dependent on the angle.

Next, we investigate the $\mathrm{O}_{2}$ molecule. Since its HOMO is degenerate and both degenerate orbitals are occupied in the ground state of the system, we calculate the momentum distribution by summing the contributions of both orbitals incoherently. Because of the cylindrical symmetry of the $\mathrm{O}_{2}$ molecule, integration over $\xi$ is unnecessary. For degenerate orbitals, there is a freedom of choice of linear combinations. We have confirmed numerically that the total momentum distribution is independent of this choice [54]. Figure 2(a) shows the contribution to the LPMD from the HOMO aligned within the plane of polarization (named $\mathrm{HOMO}_{1}$ in the following). Figure 2(b) shows the contribution of the HOMO aligned orthogonally to the polarization plane (named $\mathrm{HOMO}_{2}$ in the following). The ionization yield of $\mathrm{HOMO}_{1}$ is much larger than that of $\mathrm{HOMO}_{2}$. Furthermore, while the signal peaks at the angles $\phi=47^{\circ}, 133^{\circ}, 227^{\circ}$, and $313^{\circ}$ in Fig. 2(a), maxima appear at $\phi=90^{\circ}$ and $270^{\circ}$ in Fig. 2(b). This means that $\mathrm{HOMO}_{2}$ is ionized preferentially when the field points along the molecular axis, while $\mathrm{HOMO}_{1}$ is ionized with the highest probability when the field points through the lobes of the orbital. This behavior can be explained by the $\pi^{*}$ symmetry of the molecular orbital which leads to destructive interference at certain angles [55,56]. In Fig. 2(b), we observe a node at $k_{z}=0$. This is a consequence of the nodal plane in the orbital being aligned with the plane of polarization.

In Fig. 3, the PAD according to Eq. (5) and the width according to Eq. (6) of both orbitals are shown. In Fig. 3(a), we observe that qualitatively, there is no difference between the response of $\mathrm{HOMO}_{1}$ and the response of both orbitals

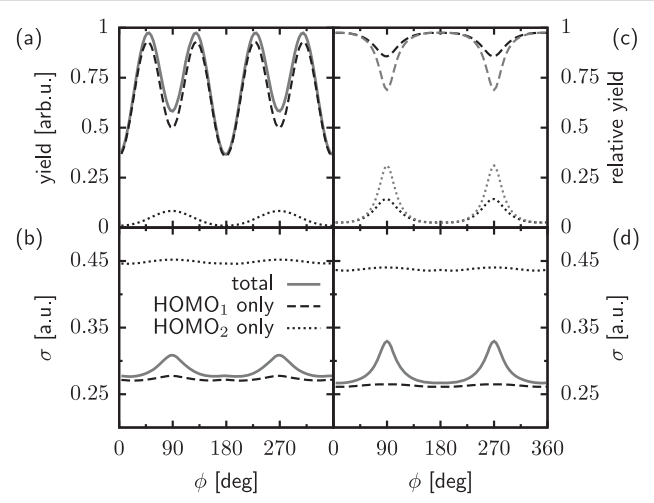

FIG. 3. (a) PAD $P(\phi)$ according to Eq. (5) using SFA for the $\mathrm{O}_{2}$ molecule ionized by an $800 \mathrm{~nm} \mathrm{cw}$ field with intensity $2.5 \times 10^{14} \mathrm{~W} / \mathrm{cm}^{2}$. Shown are curves for the response of $\mathrm{HOMO}_{1}$ only (black dashed curve), $\mathrm{HOMO}_{2}$ only (black dotted curve), and the total response of both orbitals combined (solid gray curve), respectively. (b) Corresponding lateral width $\sigma(\phi)$ according to Eq. (6) for the same PMD. (c) Contributions of $\mathrm{HOMO}_{1}$ (dashed curves) and $\mathrm{HOMO}_{2}$ (dotted curves) relative to the total angular distribution. Black curves show $800 \mathrm{~nm}$ results. Gray curves show results from an additional calculation for $1500 \mathrm{~nm}$. (d) Lateral width obtained with $1500 \mathrm{~nm}$ wavelength.

combined, since $\mathrm{HOMO}_{2}$ contributes only very weakly to the ionization yield. In contrast, the width $\sigma$ in Fig. 3(b) behaves differently. For the individual orbitals, the width is almost angle independent, but the combined response exhibits pronounced maxima at $\phi=90^{\circ}$ and $\phi=270^{\circ}$. This is due to a modulation in the relative ionization yield of the two orbitals. Around the angles $\phi=90^{\circ}$ and $\phi=270^{\circ}$, the relative contribution of $\mathrm{HOMO}_{1}$ has a local minimum, while that of $\mathrm{HOMO}_{2}$ has a local maximum, see also Fig. 3(c). $\mathrm{HOMO}_{2}$ has a much larger width than $\mathrm{HOMO}_{1}$ due to the appearance of the nodal plane at $k_{z}=0$. This results in the increase of the overall width visible in Fig. 3(c). This effect is enhanced at longer laser wavelength since the modulation depth of the relative yield becomes stronger, see Figs. 3(c) and 3(d). In a fully adiabatic picture, one expects equal contributions of both orbitals at the angles $\phi=90^{\circ}$ and $\phi=270^{\circ}$, when the field points along the molecular axis.

We calculate the PAD and the width of the LPMD for various other molecules (see Fig. 4) using the SFA. In

(a) $\mathrm{O}_{2}$ (degenerate) (b) $\mathrm{CO}_{2}$ (degenerate) (c) $\mathrm{C}_{3} \mathrm{H}_{4}$ (propadiene) (d) $\mathrm{C}_{2} \mathrm{H}_{4}$

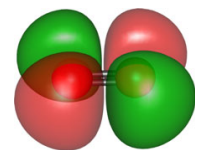

(e) $\mathrm{C}_{2} \mathrm{~F}_{4}$
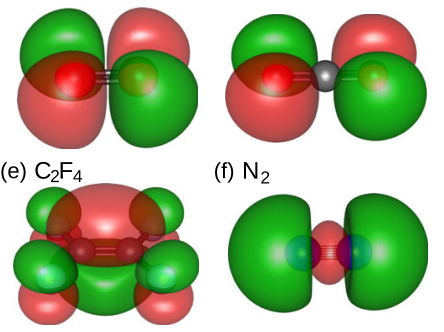

(f) $\mathrm{N}_{2}$

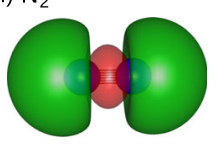

(g) $\mathrm{H}_{2}^{+}$
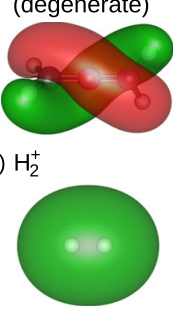

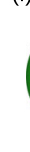

FIG. 4 (color online). (a)-(g) Overview over the HOMOs of the molecules investigated in this Letter. 


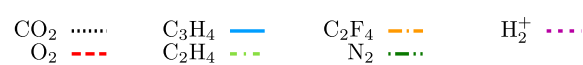

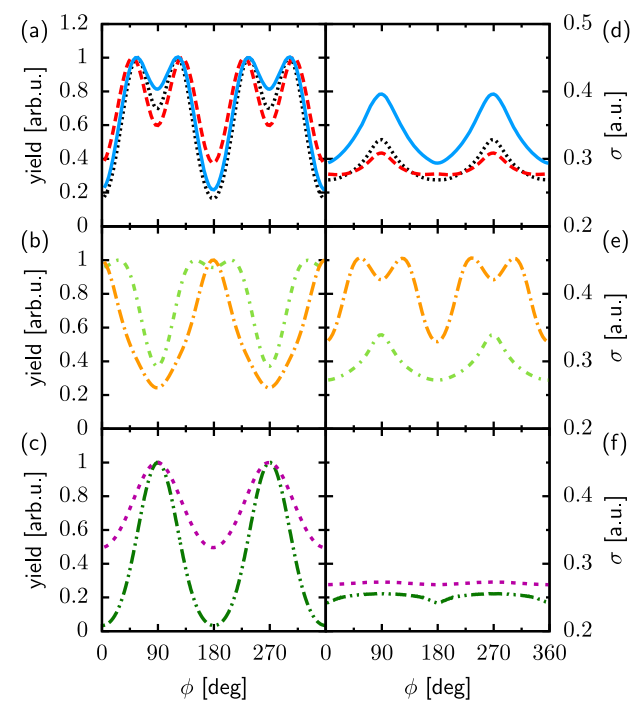

FIG. 5 (color online). (a)-(c) PADs according to Eq. (5) for various molecules using SFA for ionization in an $800 \mathrm{~nm}$ cw field with intensity $2.5 \times 10^{14} \mathrm{~W} / \mathrm{cm}^{2}$. For $\mathrm{H}_{2}{ }^{+}$, an intensity of $8.49 \times 10^{14} \mathrm{~W} / \mathrm{cm}^{2}$ is used because of the substantially larger ionization potential. Panels (d)-(f) show the corresponding lateral width $\sigma(\phi)$ according to Eq. (6).

Figs. 5(a)-5(c), the PADs are shown for ionization with an $800 \mathrm{~nm} \mathrm{cw}$ field. The width $\sigma(\phi)$ is shown in Figs. 5(d)-5(f). The curves are grouped according to the orbital symmetry: Figures 5(a) and 5(d) display the response of $\pi^{*}$-type orbitals $\left(\mathrm{C}_{3} \mathrm{H}_{4}\right.$ does not strictly belong into this category), Figs. 5(b) and 5(e) show the response of molecules with $\pi$-type HOMOs, and Figs. 5(c) and 5(f) show molecules with $\sigma$ symmetry. We observe clear differences in the PAD and in the width. Because of their orbital shape, the PADs of $\pi^{*}$-type orbitals have a maxima around $\phi=45^{\circ}$ and a strong suppression at $\phi=0^{\circ}$ and $\phi=180^{\circ}$. In contrast, the PADs of $\pi$-type orbitals exhibit a maximum at (or around) $\phi=0^{\circ}$ and $\phi=180^{\circ}$, indicating a high ionization probability when the field points orthogonally to the molecular axis. The $\pi$-type orbitals instead show suppressed ionization when the field points along the molecular axis, corresponding to deep minima in the PAD at $\phi=90^{\circ}$ and $\phi=270^{\circ}$. Concerning the width $\sigma(\phi)$, however, $\pi$ and $\pi^{*}$-type orbitals behave similarly. For both orbital symmetries, minima in the width are detected at $\phi=0^{\circ}$ and $\phi=180^{\circ}$, corresponding to ionization times where the field points orthogonally to the molecular axis. At $\phi=90^{\circ}$ and $\phi=270^{\circ}$ the width is substantially larger. This can be explained with the nodal plane of these orbitals, similarly as in $\mathrm{O}_{2}$. Although the molecules $\mathrm{C}_{2} \mathrm{H}_{4}, \mathrm{C}_{2} \mathrm{~F}_{4}$ with $\pi$-type orbitals do not have degenerate HOMOs, contributions of molecular orientations where the nodal plane lies within the plane of polarization have a similarly increasing effect on the lateral width as the degenerate HOMO in $\mathrm{O}_{2}$. In contrast to the other molecules, $\mathrm{C}_{2} \mathrm{~F}_{4}$ exhibits a double maximum in the width. This can be explained by an enhanced ionization probability for the molecular orientation with its nodal plane in congruence with the polarization plane, when the field vector points diagonally through the fluorine substituents, see Fig. 4. The width of $\sigma$-type orbitals, on the contrary, looks qualitatively different: here, the absence of a nodal plane containing the molecular axis makes the width almost independent of $\phi$, although the PAD shows a strong angle dependence. Our results suggest that a strong variation in the angle dependence of the width is an indicator of multiorbital or multiorientation contributions to the total ionization. Although typical experimental degrees of laser-induced alignment are modest (e.g., $\left\langle\cos ^{2} \theta\right\rangle \approx 0.6$ for $\mathrm{O}_{2}, \mathrm{~N}_{2}, \mathrm{CO}_{2}[57,58]$ ), the slowly varying angle dependence for $\pi$ and $\pi^{*}$-type orbitals should be measurable. Coincidence based measurements are not limited by the degree of alignment.

To conclude, we have investigated the ionization of small aligned molecules by circularly polarized laser fields. We have found evidence that the PAD in the plane of polarization as well as the LPMD carry signatures of the symmetry of the molecular orbital from which ionization takes place. The lateral width is significantly influenced by orbitals that contribute only weakly to ionization, such as $\pi^{*}$ orbitals with a nodal plane parallel to the polarization plane. In such a case, a node appears in the LPMD. This causes an increase in the overall lateral width at angles where this orbital has an increased contribution to the total ionization. This effect becomes more pronounced with increasing wavelength of the ionizing field. The increase in the width is a multiorbital effect, or similarly a multiorientation effect for the case that the molecule is not completely fixed by one-dimensional alignment. It will therefore be interesting to investigate whether lower lying orbitals can have a similar impact on the lateral width. Our calculations show that the lateral width provides information on the electronic structure that is inaccessible by measurement of the PAD alone, and furthermore that measurement of three-dimensional PMDs from aligned molecules allows us to distinguish different orbital symmetries. Although the experimental implementation is challenging, we believe that the LPMD can become an important tool in molecular imaging.

We thank the Deutsche Forschungsgemeinschaft for financial support.

[1] M. Lein, J. Phys. B 40, R135 (2007).

[2] S. Haessler, J. Caillat, and P. Salires, J. Phys. B 44, 203001 (2011).

[3] P. Salières, A. Maquet, S. Haessler, J. Caillat, and R. Taïeb, Rep. Prog. Phys. 75, 062401 (2012).

[4] M. Meckel, D. Comtois, D. Zeidler, A. Staudte, D. Pavičić, H. C. Bandulet, H. Pépin, J. C. Kieffer, R. Drner, D. M. Villeneuve, and P. B. Corkum, Science 320, 1478 (2008). 
[5] M. Lein, J. P. Marangos, and P. L. Knight, Phys. Rev. A 66, 051404 (2002).

[6] C. I. Blaga, J. Xu, A. D. DiChiara, E. Sistrunk, K. Zhang, P. Agostini, T. A. Miller, L. F. DiMauro, and C. D. Lin, Nature (London) 483, 194 (2012).

[7] H. Niikura, F. Legare, R. Hasbani, A. D. Bandrauk, M. Y. Ivanov, D. M. Villeneuve, and P. B. Corkum, Nature (London) 417, 917 (2002).

[8] A. S. Alnaser, X. M. Tong, T. Osipov, S. Voss, C. M. Maharjan, B. Shan, Z. Chang, and C. L. Cocke, Phys. Rev. A 70, 023413 (2004).

[9] J. Itatani, J. Levesque, D. Zeidler, H. Niikura, H. Pepin, J. C. Kieffer, P. B. Corkum, and D. M. Villeneuve, Nature (London) 432, 867 (2004).

[10] S. Haessler, J. Caillat, W. Boutu, C. Giovanetti-Teixeira, T. Ruchon, T. Auguste, Z. Diveki, P. Breger, A. Maquet, B. Carre, R. Taïeb, and P. Salières, Nat. Phys. 6, 200 (2010).

[11] C. Vozzi, M. Negro, F. Calegari, G. Sansone, M. Nisoli, S. De Silvestri, and S. Stagira, Nat. Phys. 7, 822 (2011).

[12] Y. Li, X. Zhu, P. Lan, Q. Zhang, M. Qin, and P. Lu, Phys. Rev. A 89, 045401 (2014).

[13] B. K. McFarland, J. P. Farrell, P. H. Bucksbaum, and M. Ghr, Science 322, 1232 (2008).

[14] O. Smirnova, Y. Mairesse, S. Patchkovskii, N. Dudovich, D. Villeneuve, P. Corkum, and M. Y. Ivanov, Nature (London) 460, 972 (2009).

[15] X. M. Tong, Z. X. Zhao, and C. D. Lin, Phys. Rev. A 66, 033402 (2002).

[16] G. Lagmago Kamta and A. D. Bandrauk, Phys. Rev. A 74, 033415 (2006).

[17] O. I. Tolstikhin, T. Morishita, and L. B. Madsen, Phys. Rev. A 84, 053423 (2011).

[18] F. Rosca-Pruna and M. J. J. Vrakking, J. Chem. Phys. 116, 6567 (2002).

[19] H. Stapelfeldt and T. Seideman, Rev. Mod. Phys. 75, 543 (2003).

[20] A. T. J. B. Eppink and D. H. Parker, Rev. Sci. Instrum. 68, 3477 (1997).

[21] M. Wollenhaupt, M. Krug, J. Khler, T. Bayer, C. SarpeTudoran, and T. Baumert, Appl. Phys. B 95, 647 (2009).

[22] J. Ullrich, R. Moshammer, A. Dorn, R. Drner, L. P. H. Schmidt, and H. Schmidt-Bcking, Rep. Prog. Phys. 66, 1463 (2003).

[23] A. Staudte, S. Patchkovskii, D. Pavičić, H. Akagi, O. Smirnova, D. Zeidler, M. Meckel, D. M. Villeneuve, R. Dörner, M. Yu. Ivanov, and P. B. Corkum, Phys. Rev. Lett. 102, 033004 (2009).

[24] X. Zhu, Q. Zhang, W. Hong, P. Lu, and Z. Xu, Opt. Express 19, 13722 (2011).

[25] M. Spanner, S. Gräfe, S. Chelkowski, D. Pavičić, M. Meckel, D. Zeidler, A. B. Bardon, B. Ulrich, A. D. Bandrauk, D. M. Villeneuve, R. Dörner, P. B. Corkum, and A. Staudte, J. Phys. B 45, 194011 (2012).

[26] L. Holmegaard, J. L. Hansen, L. Kalhj, S. L. Kragh, H. Stapelfeldt, F. Filsinger, J. Kpper, G. Meijer, D. Dimitrovski, M. Abu-samha, C. P. J. Martiny, and L. B. Madsen, Nat. Phys. 6, 428 (2010).

[27] J. L. Hansen, H. Stapelfeldt, D. Dimitrovski, M. Abu-samha, C. P. J. Martiny, and L. B. Madsen, Phys. Rev. Lett. 106, 073001 (2011).
[28] J. L. Hansen, L. Holmegaard, L. Kalhøj, S. L. Kragh, H. Stapelfeldt, F. Filsinger, G. Meijer, J. Küpper, D. Dimitrovski, M. Abu-samha, C. P. J. Martiny, and L. B. Madsen, Phys. Rev. A 83, 023406 (2011).

[29] D. Dimitrovski, M. Abu-samha, L. B. Madsen, F. Filsinger, G. Meijer, J. Küpper, L. Holmegaard, L. Kalhøj, J. H. Nielsen, and H. Stapelfeldt, Phys. Rev. A 83, 023405 (2011).

[30] M. Odenweller, N. Takemoto, A. Vredenborg, K. Cole, K. Pahl, J. Titze, L. P. H. Schmidt, T. Jahnke, R. Dörner, and A. Becker, Phys. Rev. Lett. 107, 143004 (2011).

[31] M. Abu-samha and L. B. Madsen, Phys. Rev. A 84, 023411 (2011).

[32] C. P. J. Martiny, M. Abu-samha, and L. B. Madsen, Phys. Rev. A 81, 063418 (2010).

[33] T. K. Kjeldsen and L. B. Madsen, J. Phys. B 37, 2033 (2004).

[34] M. Busuladžić, A. Gazibegović-Busuladžić, W. Becker, and D. B. Milošević, Eur. Phys. J. D 67, 61 (2013).

[35] D. Dimitrovski, C. P. J. Martiny, and L. B. Madsen, Phys. Rev. A 82, 053404 (2010).

[36] E. Hasović, M. Busuladžić, W. Becker, and D. B. Milošević, Phys. Rev. A 84, 063418 (2011).

[37] X. Zhu, Q. Zhang, W. Hong, P. Lu, and Z. Xu, Opt. Express 19, 24198 (2011).

[38] D. Shafir, H. Soifer, C. Vozzi, A. S. Johnson, A. Hartung, Z. Dube, D. M. Villeneuve, P. B. Corkum, N. Dudovich, and A. Staudte, Phys. Rev. Lett. 111, 023005 (2013).

[39] N. B. Delone and V. P. Krainov, J. Opt. Soc. Am. B 8, 1207 (1991).

[40] I. Dreissigacker and M. Lein, Chem. Phys. 414, 69 (2013).

[41] J. Henkel, M. Lein, V. Engel, and I. Dreissigacker, Phys. Rev. A 85, 021402(R) (2012).

[42] L. V. Keldysh, Sov. Phys. JETP 20, 1307 (1965).

[43] F. H. M. Faisal, J. Phys. B 6, L89 (1973).

[44] H. R. Reiss, Phys. Rev. A 22, 1786 (1980).

[45] C. Lee, W. Yang, and R. G. Parr, Phys. Rev. B 37, 785 (1988).

[46] A. D. Becke, J. Chem. Phys. 98, 5648 (1993).

[47] L. Arissian, C. Smeenk, F. Turner, C. Trallero, A. V. Sokolov, D. M. Villeneuve, A. Staudte, and P. B. Corkum, Phys. Rev. Lett. 105, 133002 (2010).

[48] P. A. Batishchev, O. I. Tolstikhin, and T. Morishita, Phys. Rev. A 82, 023416 (2010).

[49] V. N. T. Pham, O. I. Tolstikhin, and T. Morishita, Phys. Rev. A 89, 033426 (2014).

[50] M. Lein, P. P. Corso, J. P. Marangos, and P. L. Knight, Phys. Rev. A 67, 023819 (2003).

[51] J. Henkel, M. Lein, and V. Engel, Phys. Rev. A 83, 051401 (R) (2011).

[52] P. B. Corkum, Phys. Rev. Lett. 71, 1994 (1993).

[53] P. Eckle, M. Smolarski, P. Schlup, J. Biegert, A. Staudte, M. Schffler, H. G. Muller, R. Drner, and U. Keller, Nat. Phys. 4, 565 (2008).

[54] I. Petersen, dissertation Leibniz Universität Hannover, Verlag Dr. Hut, Munich, 2015.

[55] J. Muth-Böhm, A. Becker, and F. H. M. Faisal, Phys. Rev. Lett. 85, 2280 (2000).

[56] A. Jaroń-Becker and A. Becker, Laser Phys. 19, 1705 (2009).

[57] J. Itatani, D. Zeidler, J. Levesque, M. Spanner, D. M. Villeneuve, and P. B. Corkum, Phys. Rev. Lett. 94, 123902 (2005).

[58] H. J. Wörner, J. B. Bertrand, P. Hockett, P. B. Corkum, and D. M. Villeneuve, Phys. Rev. Lett. 104, 233904 (2010). 